\title{
Hétérogénéité dans des populations de tomate sous serre et répercussions sur la conduite technique. II. Effets de la conduite plante à plante sur la production de fruits
}

\author{
M Navarrete ${ }^{1 *}$, B Jeannequin ${ }^{2}$ \\ 1 INRA, systèmes agraires et développement, 16, rue Claude-Bernard, F75231 Paris cedex 05; \\ 2 INRA, systèmes agraires et développement, domaine du Mas-Blanc, F66200 Alénya, France
}

(Reçu le 4 avril 1995; accepté le 30 juin 1995)

\begin{abstract}
Résumé — L'hétérogénéité d'état végétatif à l'intérieur d'une population de tomate sous serre et en hors-sol a un effet négatif sur la qualité de la production parce qu'elle accroît la dispersion du calibre des fruits. Dans cet essai, on teste sur des placettes hétérogènes formées de l'alternance de plantes «faibles» et "vigoureuses» plusieurs traitements expérimentaux afin de réduire l'hétérogénéité du couvert. Pour cela, on cherche à favoriser la croissance végétative des plantes «faibles" en jouant soit sur l'équilibre source/puits intra-plante (ablation d'un bouquet floral), soit sur la répartition de la lumière entre plantes (effeuillage des plantes "vigoureuses", augmentation de la distance entre plantes). Ces techniques sont comparées à un traitement témoin où toutes les techniques sont appliquées de façon homogène sur la placette. Les techniques testées permettent de réduire significativement l'hétérogénéité d'état végétatif (diamètre de la tige, masse sèche foliaire) et donc la dispersion du calibre des fruits. Elles sont sans effet sur le rendement du couvert, du fait d'importants phénomènes de compensation entre plantes : les plantes "faibles", dont on a favorisé la croissance, ont une production de fruits supérieure au témoin, mais simultanément, la production des plantes «vigoureuses» est pénalisée.
\end{abstract}

Lycopersicon esculentum Mill = tomate $/$ serre $/$ techniques manuelles $/$ hétérogénéité $/$ élaboration du rendement

Summary - Heterogeneity in a glasshouse tomato crop and its effect on crop management. II. Effect on yield of adapting manual techniques plant by plant. The heterogeneity of individual vegetative states within a soilless tomato crop in the greenhouse reduces the quality of the production because the fruit grade distribution is more scattered than in an homogeneous population. We experimented several techniques in order to reduce vegetative heterogeneity. The experimental plots were established by mixing 'weak' and 'vigorous' plants. The aim of the experimental treatments was to improve vegetative growth conditions of 'weak' plants, either by altering the source/sink balance in 'weak' plants (truss pruning), or by altering the distribution of light between 'weak' and 'vigorous' plants (deleafing 'vigorous' plants, increasing plant distance). These techniques were compared with a control plot in which the cultivation technique was identical on every plant. The treatments help reduce the heterogeneity of vegetative stages (stem diameter, leaf dry weight) and mean fruit weight. They had no effect on yield per area, because of strong competition between plants: 'weak' plants had a higher fruit production than the control, but the production of 'vigorous' plants was simultaneously decreased.

Lycopersicon esculentum Mill = tomato / glasshouse $/$ manual techniques $/$ heterogeneity / yield formation

* Correspondance et tirés à part 


\section{INTRODUCTION}

Une population de tomate (Lycopersicon esculentum Mill) sous serre présente une forte hétérogénéité entre plantes (croissance végétative, composantes du rendement), qui provient pour une large part des caractéristiques de la culture : longueur du cycle (jusqu'à 11 mois, dont 6 à 7 mois de récolte), nombre élevé d'interventions manuelles sur les plantes, qui occasionnent des stress plus ou moins importants, hétérogénéité spatiale du milieu sous serre, notamment de la lumière.

Au cours d'expérimentations antérieures (Navarrete, 1994), nous avons étudié les effets de l'hétérogénéité d'état végétatif sur la production de fruits : nous avons montré qu'elle n'a pas d'effet sur le rendement du fait d'importants phénomènes de compensation entre plantes. En revanche, elle réduit fortement la qualité de la production car elle accroît la dispersion des calibres et donc la fréquence de fruits de calibres extrêmes. Comme la commercialisation des tomates de serre se fait en cagette, par calibre, l'homogénéité est un critère de qualité primordial. On retrouve des problématiques similaires pour d'autres espèces, en particulier les légumes, où la qualité est souvent déterminée par la distribution des caractéristiques individuelles des organes récoltés (par exemple la carotte: Benjamin, 1982).

La contexte économique du maraîchage sous serre est aujourd'hui difficile et il est de plus en plus nécessaire d'améliorer la qualité et de limiter les pertes. Dans certaines exploitations, on constate que des opérations techniques sont adaptées à l'état végétatif de chaque plante. $\mathrm{Ceci}$ est possible pour les opérations qui sont faites manuellement sur une plante après l'autre, comme la taille des bouquets floraux, des feuilles, des bourgeons. Les maraîchers qui font une "conduite plante à plante de la culture" cherchent ainsi à réduire l'hétérogénéité. L'analyse de cette pratique agricole originale nous semble importante (Navarrete, 1993a).

C'est pourquoi l'objectif du travail présenté ici est de répondre à 2 questions :

- la conduite plante à plante réduit-elle l'hétérogénéité d'état végétatif de la population ?

- quels sont ses effets sur la production de fruits (qualité, composantes du rendement) ?

Le principe de l'expérimentation consiste à comparer sur des placettes hétérogènes :
- un traitement témoin sur lequel toutes les techniques sont appliquées de façon identique sur toutes les plantes ;

- des traitements qui simulent des conduites plante à plante de la population, sur lesquels certaines opérations techniques sont adaptées à l'état végétatif de chaque plante.

\section{MATÉRIELS ET MÉTHODES}

L'essai réalisé sur le domaine INRA d'Alénya porte sur une culture de tomate à contre-saison et en hors-sol. Les graines (variété Capello à croissance indéterminée) sont semées le 14 novembre en pépinière, dans des bouchons de laine de roche; les plantules sont repiquées dans des cubes de laine de roche de $1 \mathrm{dm}^{3}$ de volume. Puis les plantes sont disposées dans la serre, à une densité de 2,2 plants $/ \mathrm{m}^{2}$. La culture se fait en doubles rangs orientés sud-est/nord-ouest (distance entre rangs : $50 \mathrm{~cm}$, sur le rang : $60 \mathrm{~cm}$, largeur des allées: $1 \mathrm{~m}$ ). Dans des conditions culturales classiques (température moyenne de $18^{\circ} \mathrm{C}$ ) un bouquet floral apparaît tous les 7 à 10 j et met 50 à 60 j pour arriver à maturité. La récolte s'étale sur 5 mois, de mars à juillet.

Pour ce type de culture, le fonctionnement du couvert présente les spécificités suivantes. La proximité des plantes au sein d'un double rang fait qu'il existe de fortes relations de compétition entre plantes, ce que les travaux précédents (Navarrete, 1994) ont confirmé. C'est la lumière qui est considérée comme le principal facteur de compétition, en raison de la position du cycle cultural par rapport à la saison, de l'indice foliaire élevé, et parce que l'alimentation au goutte à goutte garantit en général des conditions d'apport en eau et en éléments minéraux satisfaisantes. Les relations de compétition aérienne entre plantes sont un élément fondamental de l'élaboration de la production (Vooren et al, 1986). Par ailleurs, la production d'une plante est gouvernée par les relations de compétition pour les assimilats carbonés entre l'appareil végétatif et les fruits. En effet, sur les variétés de tomate à croissance indéterminée, un nouveau sympode apparaît chaque semaine (formé de 3 feuilles et d'un bouquet floral). Compte tenu du nombre élevé de bouquets par plante et de fleurs par bouquet, le nombre de puits potentiels (fleurs, fruits, jeunes feuilles) est très supérieur aux capacités de fabrication d'assimilats des plantes (Ho et Hewitt, 1986). À partir d'un certain degré de déséquilibre entre offre et demande en assimilats, la croissance végétative est fortement ralentie (Hurd et al, 1979) et des défauts de nouaison apparaissent (Bertin, 1994), qui déterminent pour une large part le rendement final.

\section{Traitements expérimentaux}

Les traitements expérimentaux qui simulent des conduites plante à plante ont pour objectif d'augmenter 
la croissance végétative des plantes faibles en les plaçant dans des conditions plus favorables. Les exigences de l'expérimentation et de l'analyse statistique des données conduisent à simplifier les traitements par rapport à la pratique des maraîchers : on expérimente sur des placettes où l'hétérogénéité est artificielle (c'est-à-dire formée de l'alternance de 2 types de plantes ayant des états végétatifs différents) et les techniques sont appliquées type par type et non plante par plante. On choisit donc les modalités techniques en fonction de l'état végétatif moyen du lot de plantes et non pas en fonction de l'état réel de chaque individu, ce qui est nécessaire puisque nous ne disposons pas de caractérisation suffisamment précise de l'état végétatif de chaque plante.

Le type (+) est formé d'individus plantés à la date optimale (15 janvier), qui correspond à la floraison du premier bouquet. Pour le type (-), la plantation est retardée jusqu'à la floraison du troisième bouquet (29 janvier), ce qui réduit la croissance végétative (Morgan et Clarke, 1975). Le comportement de ces 2 types a été caractérisé dans l'article précédent (Navarrete, 1994) : la vitesse de développement, la surface foliaire et le diamètre de la tige (qui en est un indicateur) sont inférieures sur le type $(-)$; de ce fait, le rendement total est réduit, ce qui est dû principalement à une diminution du poids moyen, donc du calibre des fruits. Par simplification, ce type de plantes sera parfois appelé type «faible» par opposition au type (+) dit "vigoureux".

Les placettes expérimentales se trouvent sur un seul rang du double rang, celui situé du côté sudouest. Elles sont composées de 3 plantes : une plante de type $(-)$ encadrée par 2 plantes de type (+) (fig 1 ). Les plantes de bordure (sur le rang de part et d'autre de la placette et sur le deuxième rang) sont des plantes (+) (cf dispositif détaillé dans Navarrete, 1994).

Sur le traitement témoin (TEM), toutes les techniques sont réalisées de façon uniforme sur les plantes $(-)$ et $(+)$.

Quatre traitements de conduite plante à plante sont testés. Pour augmenter la croissance végétative des plantes (-), qui sont défavorisées pour l'interception de la lumière, on agit de 2 façons, en augmentant soit les capacités d'interception de la lumière (donc la surface foliaire), soit la quantité de lumière disponible. Le choix des modalités à tester s'appuie sur la connaissance des interactions entre les techniques et l'état végétatif des plantes, grâce à la bibliographie et aux avis d'experts. Les traitements sont appliqués au cours du mois de février : pendant cette période, qui précède le début de la récolte, la charge en fruits sur les plantes est élevée, le rayonnement est faible et on observe fréquemment une diminution de la croissance végétative.

Le traitement $\mathrm{ABL}$ consiste à faire une ablation du quatrième bouquet floral sur la plante $(-)$. Cette technique est utilisée par les maraîchers en période hivernale ; on sait qu'elle modifie la répartition des assimilats carbonés et entraîne une augmentation de la surface foliaire et du poids moyen des fruits (Slack et Calvert, 1977 ; Hurd et al, 1979). L'opération a lieu avant la floraison du bouquet, le 6 février ( $84 \mathrm{j}$ après semis).
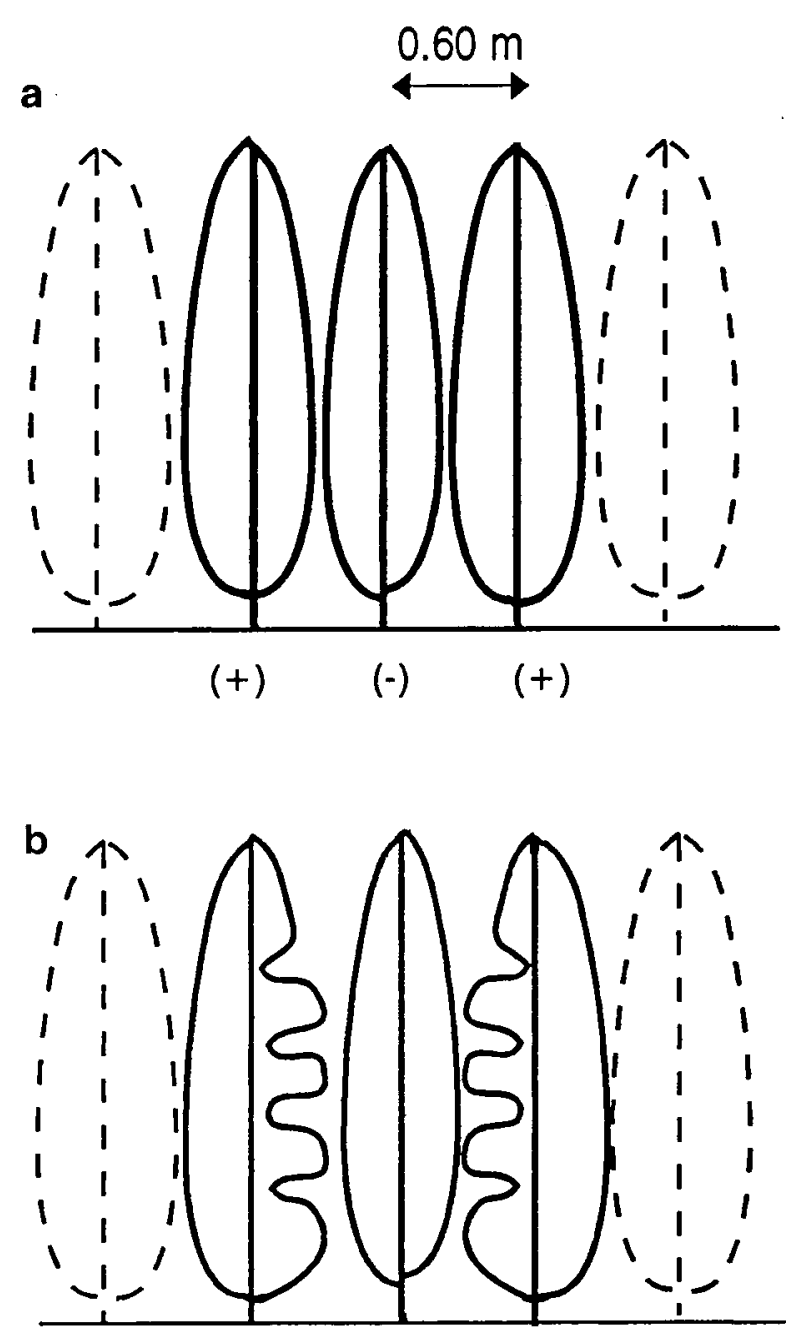

$(+) \quad(-) \quad(+)$

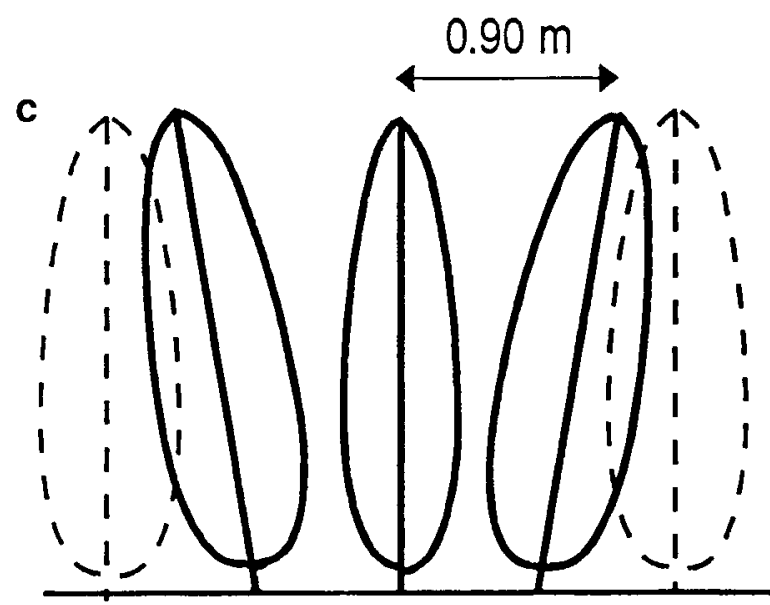

$(+) \quad(-) \quad(+)$

Fig 1. Les traitements témoin, effeuillage et distance. Vue de profil de la placette expérimentale ; les plantes de la placette sont indiquées en traits pleins, les plantes de bordure en pointillés. a Traitement témoin (TEM). b Traitement effeuillage (EFF2). Les zones incurvées sur les plantes (+) indiquent la position des feuilles supprimées $(F 13, F 15, F 17$, F19). c Traitement distance (DIST). 
Seul le traitement $A B L$ correspond à une pratique de conduite plante à plante sur les exploitations. Mais nous avons imaginé d'autres traitements, dont l'objectif est d'augmenter le rayonnement incident sur la plante faible en agissant sur les 2 plantes vigoureuses qui l'encadrent sur la placette.

Les traitements effeuillage (EFF) et distance (DIST) sont réalisés le 21 février (99 j après semis), aux environs de la floraison du bouquet B5.

Dans le traitement EFF (fig 1b), on fait l'hypothèse que l'effeuillage partiel des plantes vigoureuses permet d'augmenter le rayonnement incident sur les plantes (-) sans trop réduire la production des plantes effeuillées. Or, Slack (1986) et Wolk et al (1983) constatent que l'effeuillage partiel des plantes de tomate entraîne une diminution de rendement fonction de sa sévérité et de la position des feuilles éliminées par rapport aux bouquets : si peu de feuilles sont éliminées et en deçà des bouquets en croissance, le rendement n'est pas affecté de façon significative. Ces résultats sont établis dans le cas de l'effeuillage de toutes les plantes d'une parcelle ; nous avons supposé que le même type de relation existait à l'échelle de la plante dans une population hétérogène. Nous avons choisi 2 intensités d'effeuillage des plantes vigoureuses.

- traitement EFF1 : suppression de F15 et F18 (situées sous les bouquets B3 et B4), soit $7 \%$ de la surface foliaire totale au moment de l'effeuillage,

- traitement EFF2 : suppression de F13, F15, F17 et F19 (entre les bouquets B2 et B4), soit $14 \%$ de la surface.

Lorsque ces feuilles n'étaient pas orientées vers la plante $(-)$, ce qui aurait été contraire à l'objectif du trai- tement, nous avons choisi la feuille située juste au dessus ou au dessous. Mais cette situation s'est produite dans un petit nombre de cas (CV $<10 \%$ ).

Le traitement distance (DIST) consiste à écarter les tiges des plantes voisines $(+)$ sur le rang (fig $1 \mathrm{c}$ ), mais à densité de plantation constante. Alors que, sur le traitement témoin, l'écartement entre tiges est de 60 $\mathrm{cm}$, sur le traitement DIST, les plantes (+) après déplacement sont distantes de la plante $(-)$ de $90 \mathrm{~cm}$ au sommet de la serre. Notons que le traitement est pertinent pour étudier le comportement des individus $(+)$ et $(-)$. Mais comme il empiète sur les plantes de bordure, nous ne pourrons analyser l'effet du traitement sur le rendement du couvert (c'est le manque de place dans la serre qui nous a empêchés d'analyser plus rigoureusement cette technique).

Sur les 5 traitements, toutes les autres techniques culturales sont réalisées de façon identique sur toutes les plantes. Les bouquets sont taillés à 6 fleurs, ce qui est une pratique courante pour la variété Capello.

\section{Plan expérimental}

Le dispositif expérimental, en blocs incomplets, résulte d'un compromis entre le souhait de prendre en compte la variabilité spatiale du climat et les contraintes d'espace dans la serre. Chaque traitement est répété 5 fois. La dispositif et les conditions expérimentales sont récapitulés dans l'article précédent (Navarrete, 1994 : EXP3, tableau l et annexe).

Tableau I. Effet des traitements expérimentaux sur les plantes du type (-).

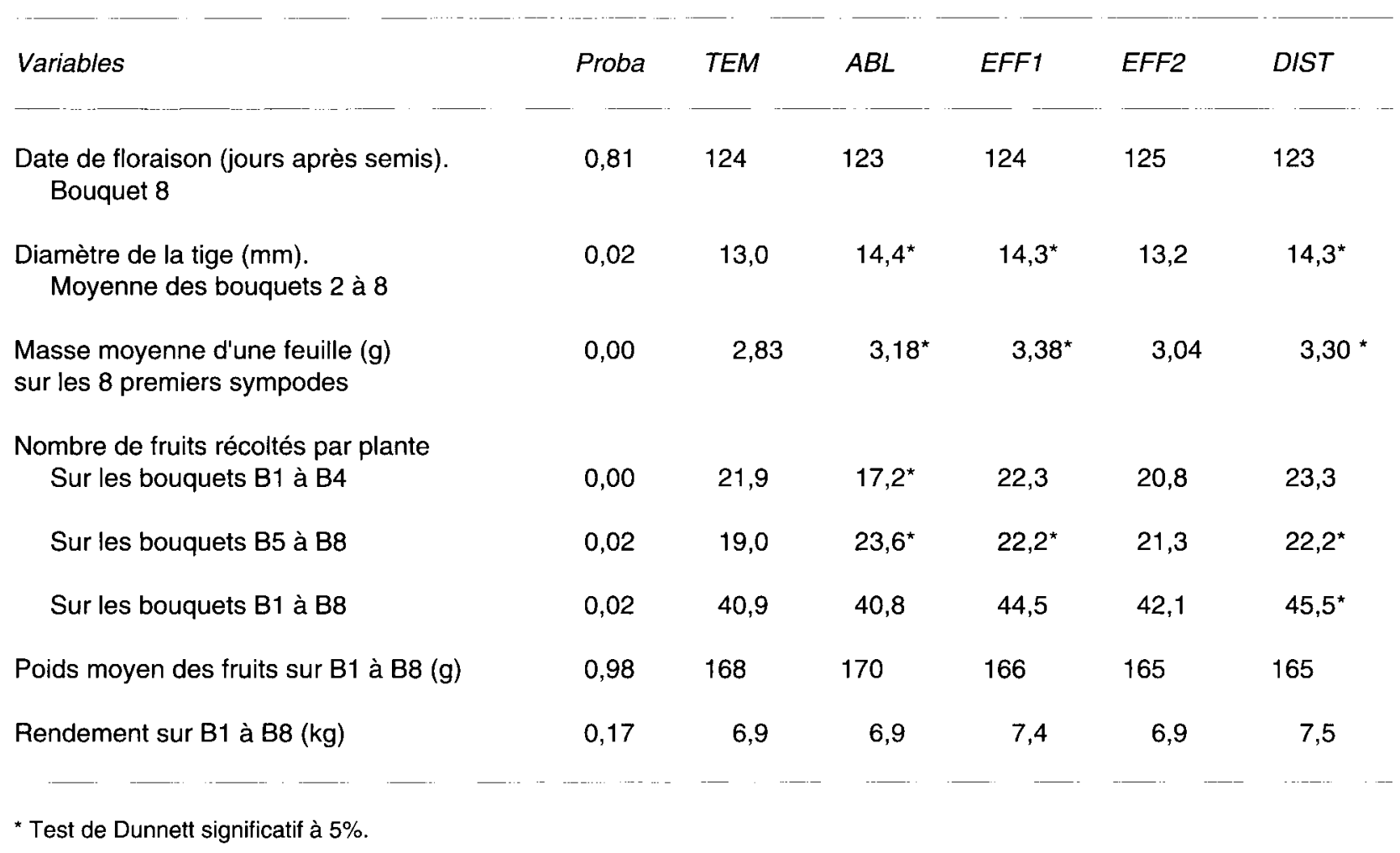




\section{Notations et mesures}

La plupart des mesures réalisées étant déjà décrites dans l'article précédent (Navarrete, 1994), nous ne faisons ici qu'un rappel. Nous avons enregistré sur les 8 premiers sympodes de chaque plante :

- un critère de développement : la date de floraison des bouquets successifs, notée lorsque la première fleur du bouquet est épanouie ;

- des mesures de l'appareil végétatif : la masse sèche des feuilles (recueillies au fur et à mesure qu'elles sont retirées de la plante) et le diamètre de la tige ;

- la production : nombre et poids de fruits récoltés 3 fois par semaine, jusqu'au 30 mai.

Les mesures effectuées visent principalement à caractériser l'effet des traitements sur les caractéristiques agronomiques des plantes. Cependant, nous avons également cherché à vérifier, par des mesures ponctuelles, si les traitements EFF et DIST permettaient bien une augmentation du rayonnement incident $\left(\right.$ PAR $_{\mathrm{i}}$ ) sur les plantes $(-)$. Une analyse plus poussée n'était pas envisageable, car le rayonnement à l'intérieur d'une culture en rang et sous serre est difficile à mesurer (Graham et al, 1990) et dépend de multiples paramètres (hauteur du soleil, heure de la journée, ombrages de la charpente, variabilité locale de l'indice foliaire...). Les modèles d'estimation du rayonnement ne sont pas utilisables dans ce cas parce qu'ils postulent une homogénéité du couvert le long du rang (Tchamitchian, 1990 ; Whitfield, 1986). Les mesures sont faites grâce à un capteur linéaire portable de 60 $\mathrm{cm}$ de longueur (ceptomètre Decagon) que l'on place horizontalement à $10 \mathrm{~cm}$ de la tige de la plante $(-)$. Elles ont lieu :

- à 4 hauteurs sur la plante $(25,50,75$ et $100 \mathrm{~cm}$ à partir du sol) ;

- dans 3 positions : perpendiculairement au rang de culture de part et d'autre de la tige $(-)$ et parallèlement au rang de culture, du côté de l'allée ;

- à 4 moments dans la journée $(9,11,13$ et 15 h) par une journée ensoleillée.

\section{Traitement des données}

Toutes les données sont enregistrées plante par plante, ce qui permet de calculer la moyenne et la variance par placette. Les données moyennes sont traitées par analyse de variance ; le test de Dunnett permet de comparer les traitements de conduite plante à plante au traitement témoin. Le test de Bartlett permet de tester l'homogénéité des variances.

\section{RÉSULTATS}

On présentera successivement l'effet des traitements expérimentaux sur les types $(-)$ et $(+)$. Puis on analysera leur effet à l'échelle de la pla- cette. Par commodité, la discussion sur l'effet des techniques d'ablation de feuilles et de fruits sur les plantes traitées sera réalisée également dans ce chapitre.

\section{Effets des traitements expérimentaux sur les 2 types de plantes}

Les traitements expérimentaux n'ont aucun effet sur la vitesse de développement, qu'il s'agisse des plantes du type (-) ou du type (+) (tableaux I et II).

\section{Traitement ablation de bouquet (ABL)}

Sur les plantes (-) du traitement ABL, c'est-à-dire celles qui ont subi l'ablation d'un bouquet (tableau I), on constate un accroissement significatif du diamètre de la tige et de la masse moyenne des feuilles sur les 8 premiers sympodes. Le nombre de fruits cumulé est significativement inférieur au témoin sur les 4 premiers bouquets, à cause de la suppression du bouquet B4. En revanche, sur les bouquets situés au-dessus du bouquet supprimé (B5 à B8), le nombre de fruits est supérieur au témoin. Comme le nombre de fleurs par bouquet est identique sur les 2 traitements, cette augmentation s'explique par une augmentation du taux de nouaison. Le nombre total de fruits récoltés sur les 8 bouquets est identique sur les 2 traitements. Comme le poids moyen des fruits est également identique sur les 2 traitements, il en est de même pour la production de fruits par plante.

Plusieurs auteurs montrent que l'ablation d'un bouquet floral entraîne une augmentation du taux de nouaison des bouquets restants et du poids moyen des fruits ; de ce fait, il n'y a généralement pas d'effet négatif sur le rendement (Slack et Calvert, 1977 ; Duyn, 1983). Ces phénomènes sont dus à une redistribution des assimilats carbonés destinés au bouquet supprimé vers les bouquets restants (Hurd et al, 1979). En particulier, comme la nouaison des fruits dépend fortement de la compétition pour les assimilats (Bertin, 1994), leur redistribution vers les jeunes bouquets explique l'augmentation du taux de nouaison sur les bouquets B5 à B8. Par ailleurs, Veliath et Ferguson (1972) observent une augmentation de la surface foliaire à la suite de la réduction du nombre de fruits. Les critères d'état végétatif mesurés (diamètre de la tige, masse foliaire unitaire) confirment que l'ablation a effectivement entraîné une augmentation de la crois- 
Tableau II. Effet des traitements expérimentaux sur les plantes du type (+).

\begin{tabular}{|c|c|c|c|c|c|c|}
\hline Variables & Proba & TEM & $A B L$ & $E F F 1$ & $E F F 2$ & $D I S T$ \\
\hline $\begin{array}{l}\text { Date de floraison (jours après semis). } \\
\text { Bouquet } 8\end{array}$ & 0,06 & 121 & 120 & 118 & 121 & 123 \\
\hline $\begin{array}{l}\text { Diamètre de la tige }(\mathrm{mm}) \\
\quad \text { Moyenne des bouquets } 2 \text { à } 8\end{array}$ & 0,00 & 16,5 & $\underset{*}{15,0}$ & 15,7 & $\begin{array}{c}14,6 \\
\star\end{array}$ & $\begin{array}{c}15,0 \\
\star\end{array}$ \\
\hline $\begin{array}{l}\text { Masse moyenne d'une feuilles ( } \mathrm{g} \text { ) } \\
\text { sur les } 8 \text { premiers sympodes. }\end{array}$ & 0,00 & 3,98 & $\underset{\star}{3,72}$ & 3,85 & $\underset{\star}{3,49}$ & $\begin{array}{l}3,62 \\
*\end{array}$ \\
\hline Masse foliaire totale $(\mathrm{g})$ & 0,00 & 97,5 & $\underset{*}{87,4}$ & 88,9 & $\underset{*}{78,0}$ & 90,8 \\
\hline Nombre de fruits récoltés par plante & 0,03 & 42,2 & $\underset{*}{45,5}$ & 43,3 & 42,6 & $\begin{array}{c}45,3 \\
*\end{array}$ \\
\hline Poids moyen des fruits $(\mathrm{g})$ & 0,04 & 184 & 170 & 183 & 161 & 175 \\
\hline Rendement (kg) & 0,04 & 7,8 & 7,7 & 7,9 & $\underset{*}{6,9}$ & 7,9 \\
\hline
\end{tabular}

Les composantes du rendement sont calculées sur les 8 premiers bouquets. ${ }^{*}$ Test de Dunnett significatif à $5 \%$.

sance végétative. L'ablation d'un bouquet a donc un effet immédiat sur les fruits (redistribution des assimilats), mais aussi un effet plus tardif dû à l'augmentation des capacités photosynthétiques des plantes.

Sur les plantes $(+)$ du traitement $A B L$, c'est-àdire les plantes voisines de celles ayant subi une ablation de bouquet (tableau II), le diamètre moyen de la tige et la masse moyenne des feuilles sont significativement inférieurs par rapport aux plantes $(+)$ du traitement témoin. Le rendement est identique au traitement témoin, mais obtenu de façon très différente : le nombre de fruits récoltés est supérieur au témoin, mais le poids moyen des fruits est inférieur.

\section{Traitements effeuillage (EFF1 et EFF2)}

Sur les plantes (+) des traitements effeuillage, c'est-à-dire celles sur lesquelles on a supprimé quelques feuilles (tableau II), on constate une réduction du diamètre de la tige et de la masse foliaire unitaire. Cette réduction est d'autant plus importante que le nombre de feuilles supprimées est élevé ; elle est significative pour le traitement EFF2. Comme le nombre de feuilles par plante sur le traitement EFF2 est réduit d'environ $14 \%$, cela fait une forte réduction de la masse foliaire totale. L'effeuillage sévère (EFF2) entraîne une forte diminution du poids moyen des fruits, donc du rendement (de l'ordre de $12 \%$ ). En revanche, l'effeuillage modéré (EFF1) n'a aucun effet sur le rendement.

L'effet de l'effeuillage sur les sympodes varie suivant leur position par rapport à la zone effeuillée : la suppression des feuilles F13, F15, $\mathrm{F} 17$ et $\mathrm{F} 19$ a un effet négatif sur la masse unitaire des feuilles situées au-dessous de la zone effeuillée (fig 2). Au contraire, les feuilles au niveau ou au-dessus de cette zone ont une

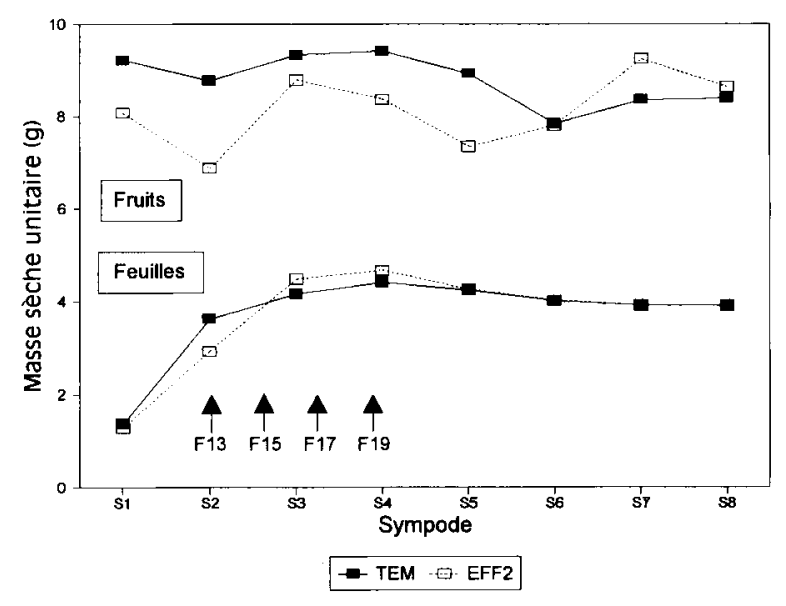

Fig 2. Effet du traitement effeuillage sévère (EFF2) sur les plantes (+). 
masse foliaire supérieure ou égale au témoin. Les bouquets les plus affectés (B1 à B5) sont ceux situés à proximité de la zone d'effeuillage.

Plusieurs auteurs (cités par Wolk et al, 1983) montrent que l'effeuillage partiel des plants de tomate entraîne une réduction des composantes du rendement (poids moyen et nombre de fruits), qui est proportionnelle à la sévérité de l'effeuillage. Pour le traitement EFF2 le plus sévère, les organes situés sous la zone effeuillée, qui étaient encore en croissance au moment de l'effeuillage, ont un poids à maturité inférieur au témoin (c'est le cas du poids moyen d'un fruit sur les bouquets $\mathrm{B} 1$ à $\mathrm{B} 5$ et de la masse sèche des feuilles $\mathrm{F} 7$ à F12). Juste après l'effeuillage, la réduction brutale de surface foliaire a réduit la photosynthèse totale, donc la croissance de ces organes. Ultérieurement, un phénomène de compensation a lieu : les feuilles F14, F16 et F18 ont une masse plus élevée que sur le témoin, ce qui indique que l'effeuillage provoque une affectation préférentielle des assimilats à l'appareil végétatif. De même, Fisher (1975) et Wolk et al (1983) mettent en évidence une augmentation de la surface des feuilles de tomate après effeuillage. Des recherches sur le haricot (Alderfer et Eagles, 1976) et sur la tomate (Tanaka et Fujita, 1974) ont montré que l'effeuillage augmente l'activité photosynthétique des feuilles restantes, afin de compenser la diminution temporaire de photosynthèse.

Sur les plantes (-) du traitement EFF2, c'està-dire les plantes voisines de celles ayant subi une ablation sévère de feuilles, le rayonnement incident est augmenté quelle que soit l'heure de la journée (fig 3). Cette augmentation est forte à

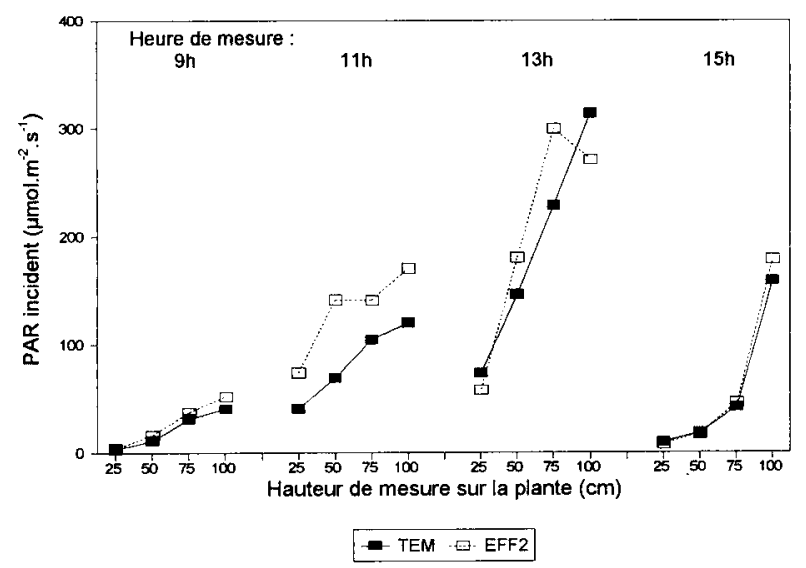

Fig 3. Effet du traitement effeuillage sévère (EFF2) sur le rayonnement incident sur la plante (-). Les mesures sont réalisées sur les placettes TEM et EFF2 d'un même bloc. Chaque point représente la moyenne de 3 répétitions. Pour le traitement EFF2, le point de mesure $13 \mathrm{~h}, 100 \mathrm{~cm}$ de hauteur est une valeur aberrante probablement due à l'ombrage d'une traverse de la charpente.
$1 \mathrm{~m}$ de hauteur, et décroît lorsqu'on s'enfonce dans le couvert. À $25 \mathrm{~cm}$ de hauteur, le rayonnement incident est généralement identique sur les traitements TEM et EFF2. Ces résultats confirment que l'effeuillage sévère des plantes $(+)$ a permis une augmentation du rayonnement absorbé par la plante $(-)$; cependant, compte tenu des facteurs de variation (heure de la journée...), il est impossible de chiffrer précisément ce phénomène. Sur le traitement EFF1, les résultats (non représentés) sont beaucoup moins nets.

Le traitement effeuillage modéré des plantes (+) entraîne une augmentation significative de 2 critères de l'appareil végétatif : diamètre de la tige et masse foliaire unitaire. La production de fruits des plantes (-) est augmentée de $7 \%$ mais l'écart n'est pas significatif. En revanche, le traitement EFF2 n'a aucun effet sur le rendement, ce qui sera discuté ultérieurement.

\section{Traitement distance (DIST)}

Rappelons que le traitement DIST consiste à éloigner les plantes $(+)$ de la plante $(-)$. Les mesures de rayonnement confirment l'augmentation du rayonnement incident sur la plante $(-)$ (fig 4) pour les 3 mesures les plus hautes $(50,75$ et $100 \mathrm{~cm}$ ) à proximité du midi solaire.

Sur ces mêmes plantes (-), le traitement DIST a un effet positif et significatif sur l'appareil végétatif de la plante (tableau I) : diamètre de tige et masse foliaire unitaire sont augmentés respectivement de 10 et $17 \%$ par rapport au témoin. Le rendement est accru de $9 \%$, mais la différence

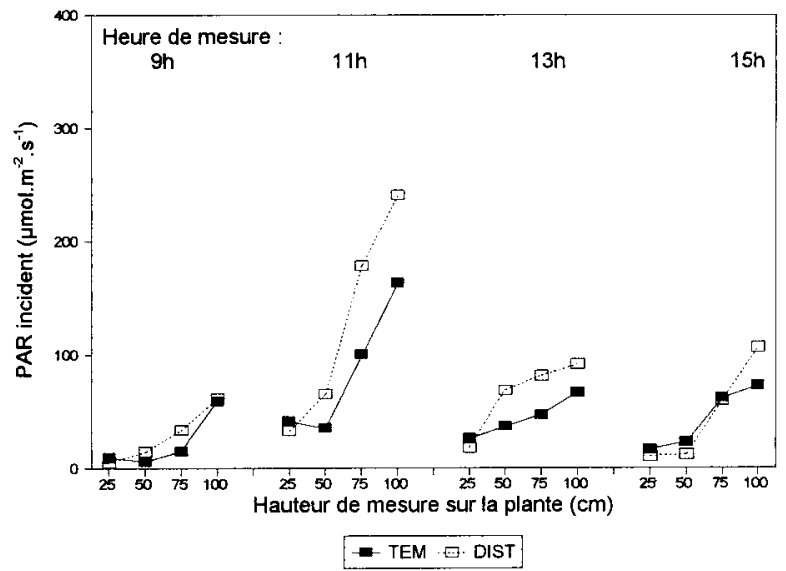

Fig 4. Effet du traitement distance (DIST) sur le rayonnement incident sur la plante $(-)$. Les mesures sont réalisées sur les placettes TEM et DIST d'un même bloc. Chaque point représente la moyenne de 3 répétitions. 
Tableau III. Effet des traitements expérimentaux sur les caractéristiques des placettes.

a. Effet sur les valeurs moyennes par placette.

\begin{tabular}{|c|c|c|c|c|c|c|}
\hline Variables & Proba & TEM & $A B L$ & $E F F 1$ & EFF2 & $D I S T$ \\
\hline $\begin{array}{l}\text { Date de floraison (jours après semis). } \\
\text { Bouquet } 8\end{array}$ & 0,19 & 122 & 121 & 120 & 123 & 123 \\
\hline $\begin{array}{l}\text { Diamètre de la tige }(\mathrm{mm}) \text {. } \\
\quad \text { Moyenne des bouquets } 2 \text { à } 8\end{array}$ & 0,10 & 15,3 & 14,7 & 15,2 & 14,1 & 14,8 \\
\hline Masse moyenne d'une feuille (g) & 0,00 & 3,60 & 3,54 & 3,69 & $3,34^{*}$ & 3,52 \\
\hline Nombre de fruits récoltés par plante & 0,01 & 41,8 & 43,9 & 43,7 & 42,4 & $45,4^{*}$ \\
\hline Poids moyen des fruits $(\mathrm{g})$ & 0,05 & 179 & 170 & 177 & $163^{\star}$ & 172 \\
\hline Rendement (kg) & 0,00 & 7,5 & 7,5 & 7,7 & $6,9^{*}$ & 7,8 \\
\hline
\end{tabular}

Les composantes du rendement sont calculées sur les 8 premiers bouquets. ${ }^{*}$ Test de Dunnett significatif à $5 \%$.

b. Effet sur les variances intra-placette.

\begin{tabular}{lcccccc}
\hline Variables & $\chi^{2}$ & TEM & ABL & EFF1 & EFF2 & DIST \\
Diamètre moyen de la tige & $17,78^{*}$ & 3,76 & 1,17 & 1,25 & 1,49 & 0,48 \\
Masse moyenne d'une feuille & $9,93^{*}$ & 0,45 & 0,12 & 0,16 & 0,19 & 0,10 \\
Nombre de fruits récoltés par plante & 1,41 & 6,35 & 6,97 & 9,49 & 6,97 & 5,06 \\
Poids moyen des fruits & $12,16^{*}$ & 458 & 144 & 253 & 71 & 0,14 \\
Rendement & 7,08 & 0,56 & 0,36 & 0,48 & 0,38 \\
\hline
\end{tabular}

* Test de Bartlett significatif à $5 \%\left(\chi^{2}\right.$ à $4^{\circ}$ de liberté $\left.=9,49\right)$.

n'est pas significative. Cette augmentation est due à un accroissement significatif du nombre de fruits récoltés sur les 8 premiers bouquets, donc du taux de nouaison.

Sur les plantes $(+)$ du traitement distance (tableau II), l'effet sur l'appareil végétatif est inverse de celui observé sur les plantes $(-)$ : diminution significative du diamètre et de la masse foliaire unitaire. Aucun effet n'est visible sur le rendement total ; cependant, le traitement joue sur les composantes du rendement : le nombre de fruits récoltés est légèrement supérieur au témoin, mais il y a simultanément une diminution du calibre des fruits.

\section{Effets des traitements expérimentaux sur la population}

À l'exception du traitement EFF2, les traitements expérimentaux qui simulent une conduite plante à plante n'ont aucun effet sur la production du couvert, aussi bien pour la production de matière sèche végétative que pour le rendement (tableau IIIa). Ceci s'explique par le fait que ces traitements entraînent simultanément une augmentation de production de matière sèche des plantes $(-)$ et une réduction des plantes $(+)$ dont les effets se compensent. En revanche le traitement EFF2 provoque une diminution significative de la 
production par unité de surface, parce que la forte diminution de production observée sur les plantes (+) effeuillées n'est pas compensée par une augmentation suffisante de production sur la plante $(-)$ voisine.

Les traitements qui simulent une conduite plante à plante entraînent une réduction significative de la variabilité intra-placette, aussi bien pour les caractéristiques de l'appareil végétatif que pour une composante du rendement, le poids moyen des fruits (tableau IIIb).

\section{DISCUSSION ET CONCLUSION}

\section{Résultats agronomiques}

Les traitements expérimentaux avaient pour objectif de réduire la variabilité d'état végétatif intra-placette. Les techniques utilisées visaient donc à placer les plantes «faibles" (petit diamètre de tige, masse foliaire réduite) dans des conditions de croissance plus favorables, l'hypothèse étant que cela entraînerait une augmentation de rendement et/ou une régulation des calibres à l'échelle de la placette. On constate effectivement une réduction de la variabilité de la matière sèche foliaire et du diamètre des tiges. De plus, les conduites plante à plante testées améliorent une composante essentielle de la qualité dans la production de tomate : l'homogénéité du calibre des fruits (en effet, il existe une bonne corrélation entre le poids d'un fruit et son calibre).

La réduction de l'hétérogénéité intiale provient à la fois d'un effet positif des traitements sur l'appareil végétatif des plantes (-), et d'un effet négatif sur les plantes (+). Pour tous les traitements, la surface foliaire des plantes (-) est accrue, mais les phénomènes impliqués sont très différents. Dans le cas de l'ablation de bouquet, on modifie l'équilibre entre appareils végétatif et reproducteur par réduction du nombre de puits ; c'est la remobilisation vers les organes végétatifs des assimilats destinés au bouquet supprimé qui permet une croissance végétatif supérieure au témoin, d'où une production de fruits supérieure (Ho et Hewitt, 1986). Dans le cas des traitements effeuillage et distance, le rayonnement supplémentaire disponible pour la plante $(-)$ est entièrement capté par ses feuilles, d'où un accroissement de la production de matière sèche, une augmentation de la surface foliaire et du rendement. Cependant, les variables disponibles ne permettent pas d'expliquer pourquoi le traitement
EFF1 (effeuillage modéré) a plus d'effet que le traitement EFF2 (effeuillage sévère) sur la production des plantes (-) alors que l'augmentation de rayonnement incident est plus forte dans le cas du traitement EFF2. Ce résultat pourrait être lié au faible nombre de répétitions par traitement (5) et à l'existence d'une variabilité intra-traitement.

Le fonctionnement de la population dépend fortement des relations de compétition entre individus, ce qui a déjà été constaté dans des recherches antérieures (Navarrete, 1994). Étant donné le mode d'action des techniques (sur les fruits, les feuilles ou la répartition du rayonnement incident), les facteurs mis en jeu traduisent probablement une compétition pour la lumière. Ceci est corroboré par le fait qu'en culture horssol l'alimentation hydrique et minérale (et notamment en azote) est rarement limitante, du moins sur une longue durée (apport de solution en excès, correction possible tout au long du cycle, faible inertie du substrat). C'est une des différences importantes avec les cultures de plein champ, où le rayonnement n'est pas le facteur limitant dans la plupart des cas.

II ne semble pas que le rendement du couvert puisse être réellement augmenté par la conduite plante à plante, mais cela mériterait d'être vérifié, en particulier pour le traitement distance : comme nous l'avions indiqué dans les Matériels et méthodes, la production de fruits des plantes de bordure, voisines des plantes (t) hors de la placette, n'a pas été enregistrée. Or, l'environnement lumineux de ces plantes étant défavorable, il est probable que leur production est diminuée, ce qui in fine pourrait se traduire par un même rendement par unité de surface que le témoin.

L'absence de différence importante sur le rendement du couvert, à la fois pour des traitements ayant des structures d'hétérogénéité différentes (Navarrete, 1994) et des conduites techniques différentes tendrait à montrer que le potentiel de la culture permis par les conditions du milieu (le rayonnement, que I'on estime être le facteur limitant) est atteint quels que soient l'état végétatif de chaque individu et les actions réalisées. En d'autres termes, pour les différents traitements étudiés, la totalité du rayonnement est captée et transformée avec la même efficience à l'échelle du couvert (en revanche, les composantes du rendement, qui conditionnent la qualité, peuvent varier grandement).

Au cours du cycle cultural de la tomate, on constate une alternance spontanée entre des périodes de forte croissance végétative et de 
faible croissance des fruits, et des périodes où les phénomènes sont inverses (De Koning, 1989). Dans cet essai, les traitements expérimentaux ont permis de modifier le classement des plantes entre elles, montrant par là même que leur état végétatif n'a jamais été définitivement affecté. La tomate est donc une espèce capable de s'adapter fortement à son environnement. Cependant, il existe probablement un seuil de vigueur à partir duquel le potentiel de croissance de la plante est définitivement réduit. Dans ce cas, il est impératif de s'interroger sur les causes de la perte de vigueur, afin d'éviter à tout prix cette situation qui ne serait pas rattrapable.

Les traitements expérimentaux choisis sont très réducteurs par rapport à la marge de manœuvre des maraîchers : nous avons appliqué la même modalité technique sur toutes les plantes d'un type alors qu'il y existe une variabilité intra-type. Or les maraîchers adaptent les opérations à l'état de chaque plante ; de plus, ils utilisent simultanément plusieurs techniques manuelles (taille des bouquets floraux, effeuillage...) et des techniques automatisées (climat, ferti-irrigation). Rappelons que l'expérimentation type par type était indispensable, puisque nous ne disposions pas d'un indicateur d'état végétatif non destructif et suffisamment fiable pour pouvoir caractériser chaque individu. Pourtant, même sans optimisation plante par plante, les effets se sont extériorisés, ce qui confirme le poids des techniques plante à plante dans l'élaboration de la production du couvert.

\section{Conséquences pour les maraîchers}

Les techniques de conduite plante à plante testées ont des conséquences agronomiques intéressantes pour les maraîchers. Elles réduisent I'hétérogénéité du calibre des fruits, ce qui présente des avantages économiques et facilite l'organisation du travail : une plus grande homogénéité des calibres permet un gain de temps au moment du calibrage et du conditionnement des fruits ; elle réduit le nombre de produits à commercialiser, donc augmente le tonnage par produit, argument commercial important sur les petites exploitations. Certaines techniques jouent aussi sur d'autres critères de qualité. Par exemple, l'ablation du bouquet permet de réduire le pourcentage de fruits de petit calibre (dont le prix de vente est faible). Cependant, l'absence d'effet négatif sur le rendement par unité de surface est une condition indispensable pour les maraîchers, car il reste le principal déterminant du chiffre d'affaires. Enfin, la conduite plante à plante, en réduisant la variabilité intra-population, pourrait résoudre certains problèmes de diagnostic, les maraîchers ne sachant pas bien définir une consigne moyenne si la variabilité entre plantes est trop forte.

Cependant, il est difficile d'évaluer la rentabilité de la conduite plante à plante d'une serre, qui résulte de l'équilibre entre coût supplémentaire de la conduite plante à plante par rapport à une conduite généralisée (augmentation des temps de travaux, du niveau de formation de la main d'œuvre) et gain attendu (en termes de valorisation de la qualité, du gain de temps au conditionnement) (Navarrete, 1993b). De plus, tous les traitements expérimentaux ne comportent pas le même risque. La technique d'effeuillage est relativement risquée, car il faut déterminer le nombre de feuilles qui permettront de maximiser l'effet sur les plantes (-) sans effet trop fort sur les plantes (+). Ce nombre est difficile à prévoir (il dépend de l'état végétatif des plantes, de la date d'intervention, des conditions du milieu et des relations de compétition entre individus) et le personnel exécutant les opérations manuelles ne dispose pas toujours du savoir-faire suffisant.

En conclusion, ces recherches permettent de discuter les pratiques observées dans les exploitations maraîchères, mais aussi d'apporter des éléments de réflexion pour l'intégration de connaissances agronomiques mises au point en parcelles homogènes sur des parcelles d'agriculteurs, qui sont fondamentalement hétérogènes.

\section{RÉFÉRENCES}

Alderfer RG, Eagles CF (1976) The effect of partial defoliation on the growth and photosynthetic efficiency of bean leaves. Bot Gaz 137, 351-355

Benjamin LR (1982) Some effects of differing times of seedling emergence, population density and seed size variation in carrot populations. J Agric Sci Camb 98, 537-545

Bertin N (1994) Competition for assimilates and fruit position affect fruit set in indeterminate greenhouse tomato. Ann Bot (sous presse)

Duyn (Van) P (1983) Truss pruning and truss removal. Groenten En Fruit 38, 32-33

De Koning ANM (1989) The effect of temperature on fruit growth and fruit load of tomato. Acta Hort 248, 329-336

Fisher KJ (1975) Effect of the amount and position of leaf tissue on the yield or single-truss tomatoes. Scientia Hortic 3, 303-308 
Graham MED, Dube PA, Phenix M (1990) Measurement of natural irradiance in greenhouses: the effect of averaging period and number of sensors on measurement reliability. Biotronics 19, 83-91

Ho LC, Hewitt JD (1986) Fruit development. In : The Tomato Crop. A Scientific Basis for Improvement (JG Atherton, J Rudich, eds), Chapman and Hall, Londres, Royaume-Uni, 201-240

Hurd RG, Gay AP, Mountifield AC (1979) The effect of partial flower removal on the relation between root, shoot and fruit growth in the indeterminate tomato. Ann App/ Biol 93, 77-89

Morgan JV, Clarke EL (1975) Influence of stage of development at planting on flowering and fruiting in tomato. Acta Hortic 51, 131-145

Navarrete M (1993a) Variabilité au sein d'un peuplement de tomate sous serre et répercussions sur la conduite technique. Analyse expérimentale et étude des pratiques culturales des maraîchers. Thèse université d'Orsay, $152 p+$ annexes

Navarrete M (1993b) L'organisation du travail, déterminant de la conduite technique d'une culture de tomate sous serre. CR Acad Agric Fr 79, 107-117

Navarrete M (1994) Hétérogénéité dans des populations de tomate sous serre et répercussions sur la conduite technique. I. Effets de l'hétérogénéité sur la production de tomate. agronomie 14, 649-660
Slack G (1986) The effects of leaf removal on the development and yield of glasshouse tomatoes. J Hortic Sci 61, 353-360

Slack G, Calvert A (1977) The effect of truss removal on the yield of early sown tomatoes. J Hortic Sci 52 , 309-315

Tanaka A, Fujita K (1974) Nutrio-physiological studies on the tomato plant. IV. Source-sink relationships and the structure of the source-sink unit. Soil Sci Plant Nutr 20, 305-315

Tchamitchian M (1990) Photosynthèse d'une culture de tomate sous serre : mise au point et validation d'un modèle analytique. Thèse doct ing INP, Toulouse, $97 \mathrm{p}$

van de Vooren J, Welles GWH, Hayman G (1986) Glasshouse crop production. In : The Tomato Crop. A Scientific Basis for Improvement (JG Atherton, J Rudich, eds), Chapman and Hall, Londres, Royaume-Uni, 581-624

Veliath JA, Ferguson AC (1972) The effect of deblossoming on fruit size, yield and earliness in tomato. Hortic Sci 7, 278-9

Whitfield DM (1986) A simple model of light penetration into row crops. Agric For Meteorol 36, 297-315

Wolk JO, Kretchman DW, Ortega DG (1983) Response of tomato to defoliation. J Am Soc Hortic Sci 108, 536-540 